\title{
FEMINISMO COMUNITARIO: PLURALIZANDO EL SUJETO Y OBJETO DEL FEMINISMO
}

\section{Community feminism: pluralizing the subject and object from feminism}

\author{
Julieta Evangelina Cano \\ cano.juieta@gmail.com \\ Universidad Nacional de La Plata - Argentina
}

Recibido: 27-01-2017

Aceptado: 05-05-2017

\section{Resumen}

La intención del presente trabajo es pensar al feminismo como un movimiento social, dentro del cual surgen otros movimientos que además de cuestionar al Patriarcado, cuestionan al propio feminismo por entender que cierto feminismo blanco, burgués y de clase media hegemonizó las demandas del colectivo, invisibilizando las situaciones de muchas mujeres con realidades específicas y demandas particulares, que no se sienten representadas, como el feminismo afrodescendiente, indígena, lesbiano, etcétera. Puntualmente me interesa abordar al feminismo comunitario para preguntarme acerca de su potencial disidente. En esta (pseudo)ruptura, aunque la identidad de lo que entendemos por feminismo no se pone en cuestión, lo cierto es que el feminismo hegemónico no estaría ofreciendo lugar para todas aquellas identidades de mujeres que necesitan crear otros espacios de identificación, sin divorciarse plenamente del feminismo, "conservando el apellido".

Palabras Clave: Feminismo hegemónico, feminismo comunitario, movimientos sociales, disidencia.

\begin{abstract}
The aim of the present paper is to think feminism as a social movement, in which other movements arise questioning not only the Patriarchy, but also questioning feminism itself. This questioning to feminism is due by the fact that white, bourgeois and middle class feminism hegemonized the demands of women's collective, making invisible the situations of many women with specific realities and particular demands, who do not feel represented as Afro-descendant feminism, Indigenous feminism, Lesbian feminism, etcetera. I am interested in exploring the dissident potential of community feminism. In this (pseudo) rupture, although the identity of what we understand as feminism is not challenged, it is true that hegemonic feminism would not be giving place to all the identities of women who need to create other spaces of identification without fully divorcing from the feminism, in other words, "preserving the surname".
\end{abstract}

Keywords: Hegemonic feminism, community feminism, social movements, dissidence. 
"Pedir al Estado el reconocimiento de los derechos civiles de la mujer y la reivindicación del niño, es tarea vana que realizan las mujeres que creen cándidamente que por medio de la ley y de la acción legalitaria (sic), conquistarán la reivindicación de estos derechos [...] Además, que pedir justicia y derechos al Estado, institución históricamente coercitiva, implica un desconocimiento absoluto de su rol de tiranía y sojuzgador de las libertades, tanto del hombre como de la mujer" (Juana Rouco, 1923)1.

\section{El paradigma de análisis de los movimientos feministas y de mujeres}

Originalmente, los movimientos feministas y de mujeres fueron conceptualizados como parte de los "nuevos movimientos sociales" (NMS), categoría surgida en los años '80 para denominar a aquellos movimientos que no tenían como actor clave al movimiento obrero (Rubio García, 2004; Parra, 2005; Viguera 2009) no siendo la clase social el eje articulador de las demandas ni de la movilización ${ }^{2}$ en un contexto en que se consideraba que los partidos políticos no ofrecían respuestas satisfactorias (Revilla Blanco, 1996). Los NMS promueven de esta manera la organización de la ciudadanía y convierten a la sociedad civil en un nuevo terreno para la lucha política, despojándola de la neutralidad asignada por el liberalismo (Arditi, 2004):

"Los "nuevos movimientos sociales" eran disruptivos, pero lejos de responder intuitivamente a situaciones críticas, lo que hacían era poner en la agenda política reclamos vinculados a clivajes que si no eran nuevos, hasta entonces no habían sido el eje de movilizaciones masivas con programas que alcanzaban altos niveles de convocatoria" (Viguera, 2009: 9).

El paradigma de los NMS se explica a través de la noción de identidad: "[s]e hace énfasis en el proceso por el cual los actores luchan por constituir nuevas identidades como medios para crear espacios democráticos y para crear una acción autónoma. Su análisis recae sobre todo en los actores y en la acción colectiva" (Parra, 2005: 76). De la misma manera, Ramos Rollón (1997) y Revilla Blanco definen a la identidad como constitutiva de los movimientos sociales: "[p]lanteamos como primera aproximación al tema la definición del movimiento social como «el proceso de (re)constitución de una identidad colectiva, fuera del ámbito de la política institucional, por el cual se dota de sentido a la acción individual y colectiva»" (Revilla Blanco, 1996: 1).

Pienso al feminismo como movimiento social que articula principalmente a mujeres en torno a una demanda por la identidad, en donde además se propone una resignificación de ese ser-mujer (Forez Florez, 2005). Uno de los objetivos de los movimientos sociales es el cuestionamiento y reforma del orden político vigente (Tapia, n.d.). En este sentido, el feminismo o los feminismos

\footnotetext{
${ }^{1}$ Citada por Valobra (2015: 32).

2 "Esto, sin embargo, no implica la existencia de una base social de los movimientos indiferenciada en términos de clase o exenta de condicionantes ideológicos, sino que, simplemente, no son éstos los determinantes que llevan a la movilización, en contraposición especialmente con el movimiento obrero" (Rubio García, 2004: 19).
} 
tienen la capacidad de presentar alternativas al orden vigente, capacidad de "[...] convencer al estado y a la sociedad civil de la posibilidad de hacer, organizar, dirigir y vivir las cosas de otro modo; la capacidad ya desarrollada por el movimiento para pasar de la crítica a la reorganización de las cosas" (Tapia, n.d.: 4).

La intención del presente trabajo es pensar al feminismo como un movimiento social, dentro del cual surgen otros movimientos que además de cuestionar al Patriarcado ${ }^{3}$, cuestionan al propio feminismo por entender que cierto feminismo blanco, burgués y de clase media hegemonizó las demandas del colectivo, invisibilizando las situaciones de muchas mujeres con realidades específicas y demandas particulares, que no se sienten representadas; como el feminismo afrodescendiente, indígena, lesbiano, etcétera. Puntualmente me interesa abordar al feminismo comunitario para preguntarme acerca de su potencial disidente. En esta (pseudo)ruptura, aunque la identidad de lo que entendemos por feminismo no se pone en cuestión, lo cierto es que el feminismo hegemónico no estaría ofreciendo lugar para todas aquellas identidades de mujeres que necesitan crear otros espacios de identificación, sin divorciarse plenamente del feminismo, "conservando el apellido".

Tal como lo plantea Revilla Blanco, este podría ser un caso en donde se pone en cuestión el orden de preferencias definido por el feminismo hegemónico como una causa de disonancia al interior del movimiento:

“[...] las expectativas de acción que corresponderían a mi potencial inscripción en un determinado grupo de reconocimiento existente no concuerdan con el orden de preferencias al que doy prioridad. En este caso, las identidades colectivas existentes no permiten a un individuo reconocerse y ser reconocido. El movimiento social resultante de este tipo de incertidumbre tenderá a constituirse como una identidad colectiva nueva" (Revilla Blanco, 1996: 10).

Tal como lo ilustra la autora, en el caso del feminismo comunitario, tenemos una doble rebelión coral ${ }^{4}$. Si el feminismo ya planteaba una rebelión del coro por la reducción de sus expectativas de acción frente a organizaciones que entendían la situación de las mujeres como no

\footnotetext{
${ }^{3}$ Heidi Hartmann define al patriarcado como "[...] un conjunto de relaciones sociales que tiene una base material y en el que hay unas relaciones jerárquicas y una solidaridad entre los hombres que les permiten dominar a las mujeres. La base material del patriarcado es el control del hombre sobre la fuerza de trabajo de la mujer" (Hartmann, 1980: 97).

${ }^{4}$ La autora explica de qué se trata la rebelión del coro, citando una metáfora de Nun: "En términos de la metáfora de Nun sobre la rebelión del coro, parte del coro se rebela cuando la tragedia que se representa en el centro del escenario no es la que quieren representar (modificación en las preferencias), o cuando se saca al coro fuera del escenario (reducción de las expectativas). En ambos casos, parte del coro demanda el cambio de tragedia y un papel que suponga dejar de ser coro" (Revilla Blanco, 1996: 14). Reproduzco a continuación la nota al pie Nro. 44 en donde explica la metáfora de Nun: "«En la tragedia griega el centro del escenario lo ocupaban casi siempre los héroes, únicos que se hallaban en contacto directo con los dioses. La vida cotidiana tenía reservado, en cambio, un espacio subalterno y sin rostro: el del coro. Lo formaban las mujeres, los niños, los esclavos, los viejos, los mendigos, los inválidos, en una palabra, todos los que se quedaban en la ciudad cuando los demás partían en busca de la aventura, del poder y de la gloria» (Nun, 1989: 11)" (Revilla Blanco, 1996:14).
} 
prioritaria, el feminismo comunitario (como tantos otros) se rebela porque sus preferencias no encuentran lugar en el accionar del colectivo ya rebelado.

\title{
2. La relación de los feminismos y el Estado
}

Considero importante reflexionar previamente al tema que me convoca, sobre la relación de los feminismos con el Estado, dado que los Movimientos Sociales necesariamente articulan con el Estado, ya sea denunciando su accionar, reclamando el reconocimiento de derechos, demandando políticas públicas e incluso considerándolo como una herramienta activa del Patriarcado para perpetuar la opresión.

Al respecto, el feminismo occidental ha tenido posiciones contrapuestas. Por un lado, pensando al Estado como un terreno más de lucha feminista y convirtiéndolo en un interlocutor válido a quien dirigir las demandas del colectivo, pero también pensándolo como el garante de las relaciones patriarcales de asimetría entre mujeres y varones. El Estado como garante remite a la manera que O’Donnell piensa el Estado: no es un Estado al servicio de los capitalistas, sino un garante de las relaciones de poder capitalistas ${ }^{5}$. Al respecto, Valobra reflexiona:

\begin{abstract}
“Así, el Estado no sólo se define por los intereses que sirve o por quiénes detentan el poder sino porque está garantizando ciertas relaciones sociales que, además de vehiculizar la existencia de ciertos modos de producción capitalistas, también supone relaciones generizadas de subordinación que impiden impugnar el sistema. Según MacKinnon, la trampa es que el Estado "protege el poder masculino" y garantiza el control de éste "sobre la mujer en todos los ámbitos" de modo que cuando el Estado brinda derechos, en realidad, no está más que "apareciendo de iure para prohibir sus excesos cuando es preciso para su normalización. Las relaciones de iure estabilizan las relaciones de facto”. Así, coincide con Pateman cuando confirma que las leyes son vehículos de la opresión porque el Estado se construye como un garante de la violencia heterosexista [...]" (Valobra, 2015: 39).
\end{abstract}

En definitiva, la relación del movimiento feminista con el Estado es compleja. Principalmente porque no existe consenso sobre el rol que tiene el Estado en relación con las demandas de las mujeres, es decir: "[s]i el Estado es un actor que se define por su punto de vista masculino y la masculinidad se define, a su vez, por la violencia que puede ejercer sobre el cuerpo de las mujeres, entonces, no hay opción y la única respuesta posible es la destrucción del Estado" (Valobra, 2015:49). Por otro lado, los avances de las mujeres en el siglo pasado han tenido como

\footnotetext{
5 A partir de caracterizar al Estado-garante, O’Donnell intenta tender un puente entre la dominación económica y la política, para pensar que Estado y Sociedad tienen vínculos, y no puede pensárselos escindidamente, porque "El Estado garantiza y organiza la reproducción de la sociedad que capitalista porque se halla respecto de ella en una relación de "complicidad estructural" (1978: 14). Sienta de esta manera, la complicidad estructural del Estado con la burguesía o con fracciones dentro de esa clase.
} 
garante al Estado, quien, aunque a regañadientes, ha reconocido paulatinamente las demandas de las mujeres en relación son sus derechos.

Dentro de esta situación en que los feminismos se debaten acerca de qué lugar darle a la intervención estatal, dentro del propio feminismo surgen corrientes que además de cuestionar al Patriarcado, al Estado Patriarcal y a sus vinculaciones con el capitalismo, en el marco de todas estas definiciones, ciertos feminismos cuestionan al feminismo que identifican como hegemónico. Dicho feminismo, blanco, occidental, burgués, invisibiliza otras marcas de subalternidad en donde la palabra "mujer" no es representativa de un colectivo tan heterogéneo como son las mujeres (en plural).

\section{Feminismo comunitario como feminismo disidente}

"No podemos entender Abya Yala y a sus mujeres si no conocemos sus luchas y sus procesos de transformación, en un momento efervescente de su historia” (Robles Santana, n.d.: 3).

Planteo al feminismo comunitario como un feminismo disidente, dada su denuncia frente al feminismo occidental hegemónico que ha obturado ciertas voces en la construcción de sus demandas, rescatando ciertas observaciones que se vienen haciendo desde varios colectivos de mujeres: lesbianas, afrodescendientes, campesinas, etcétera: “[s]e abren así viejas discusiones en torno a quién puede hablar por otros y de qué modo algunas feministas están monopolizando la representación y borrando las marcas intragenéricas, etáreas, de raza, entre otras, tal como ya lo denunciaran las feministas negras y lesbianas en los 70 o, más recientemente, las del tercer mundo" (Valobra, 2015: 47). La ruptura epistemológica con el feminismo occidental por parte del feminismo comunitario, empieza con una definición: “[n]os parece importante partir de nuestra definición de feminismo: feminismo es la lucha y la propuesta política de vida de cualquier mujer en cualquier lugar del mundo, en cualquier etapa de la historia que se haya rebelado ante el patriarcado que la oprime" (Paredes, 2014: 76). A partir de esta definición, el feminismo comunitario se define como feminismo, y reconoce interlocutores en otros feminismos que articulen luchas contra el Patriarcado. Por ello es disidente, porque es feminismo, porque no desconoce al feminismo blanco y burgués, sino que no se identifica con los postulados del mismo, no se siente representado. Hay una reformulación identitaria dentro del propio movimiento social que no cuestiona la identidad matriz, pero que la complejiza.

El feminismo comunitario contribuye a ampliar el sujeto y el objeto de los feminismos, y ayuda también a pensar desde los feminismos (plural) y no desde un feminismo occidental y hegemónico que tiene a una sujeta política a veces bien diferente de la reivindicada desde el feminismo comunitario. La intersectorialidad es parte constitutiva de este movimiento epistémico diferente: el feminismo comunitario, que tiene raíz indígena, rescata las luchas ancestrales de las tatarabuelas (con intención de descolonizar la categoría género), y se presenta como la contracara 
del feminismo hegemónico porque no plantea una confrontación intergéneros por los derechos individuales, sino que afirma el ser-mujer desde la comunidad, vista ésta como un cuerpo en donde varones y mujeres no podrían escindirse.

Este feminismo denuncia la existencia de un Patriarcado ancestral y originario, como causante de la ruptura entre varones y mujeres. Julieta Paredes, referente del feminismo comunitario boliviano, plantea que los lineamientos de políticas públicas, desde la matriz del feminismo comunitario, deben inscribirse en 5 campos de lucha y acción: cuerpo de las mujeres, espacio de las mujeres, movimiento organizativo y autónomo de las mujeres, memoria (reconociendo un Patriarcado pre-colonial) y tiempo de las mujeres (Paredes, 2014).

El feminismo comunitario propone un marco conceptual dinámico en donde se identifican cinco campos de acción y de lucha de las mujeres desde las comunidades. La primera dimensión es la del cuerpo y el reconocimiento de la existencia de cuerpos sexuados y atravesados por otras diferencias: color de piel, estatura, peso, etcétera. El feminismo comunitario concibe a las personas como una unidad, no abonando la dicotomía mente-cuerpo. Una de las demandas sostenidas desde este feminismo es la de lograr la representación política con nuestros cuerpos de mujeres (Paredes, 2014), visibilizando las diferencias, pero para combatir las desigualdades que sobre ellas se instauraron.

El espacio aparece como otra de las dimensiones, y es el lugar en donde el cuerpo se desarrolla. La demanda por el espacio para las mujeres comprende no sólo el reconocimiento en el territorio, sino también el acceso a la vivienda y a los recursos naturales, y el tránsito por el mismo -entre otras-. El tiempo está pensado desde una concepción cíclica, recuperando los puntos en común de las mujeres y la naturaleza, pero además revalorizando el tiempo usado por las mujeres como tiempo importante: "[e]n nuestra concepción, la cotidianeidad y lo llamado histórico son un continuum, son parte de la vida que se alimenta" (Paredes, 2014: 111).

La revalorización del trabajo doméstico, pero además el permitir el uso del tiempo para cosas diferentes al mismo, como estudiar o descansar son parte de las reivindicaciones. La cuarta dimensión de las reivindicaciones y resignificaciones que se proponen desde el feminismo comunitario tiene que ver con el movimiento y los procesos que se dan en el mismo. El movimiento se relaciona con contar con organizaciones propias para las mujeres en donde pueda operar la representación y autorepresentación y la forma horizontal de organización, como también la posibilidad de alianzas con otras organizaciones de mujeres. Por último, la dimensión de la memoria, pero no para remontarse a los tiempos precoloniales construyendo un momento idílico de relaciones entre varones y mujeres: "[h]ay que despatriarcalizar la memoria y reconocer que hubo un patriarcado precolonial y que la situación de las mujeres no es sólo a partir de la colonia y la llegada de los españoles" (Paredes, 2014: 117). En el recuperar la memoria se pretende, sobre todo, recuperar y revalorizar las sabidurías de las mujeres y su derecho a la producción de conocimiento.

El feminismo comunitario parte de otra concepción de la vida y de la comunidad, como un todo integrado separado de los dualismos jerarquizados, reivindica el análisis intersectorial, 
transformando a la mujer en las mujeres, yendo más alá de todo concepto esencialista partiendo de la diversidad femenina existente en Abya Yala ${ }^{6}$. El feminismo comunitario, además, se inscribe en una corriente de producción de saberes desde el sur, o como lo plantea Forez Florez:

“Como veremos más adelante, sus ejercicios ya no serían «sobre» la periferia, sino «desde» la periferia (entendida como postura epistémica fronteriza). Por ejemplo, ciertas investigaciones Feministas ofrecen sólidos argumentos para entender cómo los movimientos de mujeres del Sur -respondiendo a la crisis de los mecanismos convencionales de representación política- amplían el carácter democrático de las prácticas sociales que definen la ciudadanía (Dagnino, 2001; Schild, 2001) y en consecuencia, redefinen los parámetros de «autonomía» frente al Estado" (Forez Florez, 2005: 79).

Jerarquizar la producción de saberes desde el Sur se relaciona con las posturas decoloniales que plantean que la modernidad de inicia en 1492 con el "descubrimiento" de América, postulando que en Latinoamérica no conocemos otro tipo de producción o reflexiones que no sean modernas ${ }^{7}$.

La premisa del feminismo comunitario es que las mujeres somos la mitad de todo ${ }^{8}$, intentando problematizar aquello que se considera "problema de mujeres" o "tema de mujeres":

"Otra forma de ejemplificar esto es cuando se habla de qué se va a tratar el tema del transporte, la seguridad ciudadana, la economía, los recursos naturales y el tema de las mujeres, como si nosotras como mujeres no tuviéramos que ver con el tema del transporte, la seguridad ciudadana, la economía y los recursos naturales" (Paredes, 2014: 48).

El feminismo comunitario entiende que el principal problema de las mujeres debido a su inscripción genérica, es que las decisiones sobre nuestros cuerpos no nos pertenecen, "siendo colonia de los hombres, los gobiernos y los Estados" (Paredes, 2014: 39). Ésta, podemos decir que es una lucha compartida y transversal a todos los movimientos feministas, es decir que no se trata de una postura disidente. La disidencia propia del movimiento dentro del movimiento se

\footnotetext{
6 “Abya Yala, palabra del pueblo indígena Kuna de Panamá que designa al territorio de América” (Robles Santana, n.d.: 1).

7 "Pero lo cierto es que, desde su misma constitución [la modernidad], ese proceso involucró a las periferias coloniales. Siendo así, ni siquiera vale la pena preguntarse si los movimientos periféricos han alcanzado o no las metas ilustradas. Latinoamérica ha sido parte de la modernidad desde el momento mismo de su constitución; es más, la posición periférica que jugó al inicio de la conformación del Sistema-Mundo fue crucial para la emergencia y posterior consolidación de la racionalidad moderna" (Forez Florez, 2005: 88). 8 "Las mujeres somos más de la mitad de la población boliviana y no somos ni una minoría, ni un tema a tratar, ni un sector, ni un problema; las mujeres somos la mitad de todo" (Paredes, 2014: 49).
} 
relaciona con el neoliberalismo ${ }^{9}$ y cómo este se articula con características propias en Latinoamérica, asumiendo el Patriarcado características coloniales y neoliberales:

\begin{abstract}
"Tenemos que reconocer que hubo históricamente un entronque patriarcal entre el patriarcado precolonial y el occidental. Para entender este entronque histórico entre los intereses patriarcales, nos es útil recuperar la denuncia del género para descolonizarlo en su entendido que las relaciones injustas entre hombres y mujeres sólo fueran fruto de la colonia, y superarlo, como concepto ambiguo fruto del neoliberalismo. Superarlo, decíamos, en la reconceptualización que desde el feminismo comunitario hoy hacemos, y trascenderlo como tarea revolucionaria a la que hoy el proceso de cambios nos convoca, especialmente a las mujeres. Para recuperar al género aparte de desneoliberalizarlo, que es lo que planteábanos en los puntos anteriores, hay que también descolonizarlo, esto porque no hay otro concepto en la actualidad que haya develado mejor la situación y condición de las mujeres como el género. Ojo, no la equidad de género sólo la denuncia del género” (Paredes, 2014: 71-72).
\end{abstract}

Al feminismo occidental le reprochan a su vez que desvirtuó la categoría género, originalmente de denuncia, y hoy funcionando como una dimensión que justifica la aplicación de políticas neoliberales (Paredes, 2014). Esto se traduce en que las políticas públicas que abogan por la igualdad de género, en una estrategia de vaciamiento del contenido político del concepto, son contradictorias en el origen, dado que el género debería dejar de existir porque se funda sobre la desigualdad:

"Para que comprendamos mejor esta explicación, queremos comparar el valor político de la categoría género con la de clase. El género tiene como valor político lo mismo que la clase: nunca va a haber equidad (igualdad) de clase, porque las clases sociales se fundan, se originan en la injusticia de la explotación de una clase sobre la otra, los burgueses son burgueses porque explotan a los proletarios [...] Lo mismo sucede con el género: nunca va a haber equidad de género entendida como igualdad, porque el género masculino se construye a costa del género femenino, por lo que la lucha consiste en la superación del género como injusta realidad histórica. Dicho de otra manera, de lo que se trata es de trascender el género, como construcción histórica y cultural y empezar una nueva forma de criar y socializar a las wawas sin géneros. Lo que se quiere desde el feminismo es ya no ser más ni femeninas ni masculinos. Queremos acabar con las relaciones de poder construidas por el género y no conservar el género en una equidad contrarrevolucionaria" (Paredes, 2014: 65-66).

Además de pensar al varón y a la mujer como seres complementarios (no en el sentido de pareja heterosexual, sino en sentido comunitario, ambos necesarios para crear comunidad):

\footnotetext{
9 "El neoliberalismo desplegó una gran propaganda posmoderna de promoción de los derechos humanos: supuestamente las mujeres, los indígenas, los homosexuales, los jóvenes, los discapacitados eran reconocidos y se les daban dizque derechos. En la realidad, sólo premiaban o reconocían a las mujeres, indígenas, homosexuales, jóvenes y discapacitados que eran de su misma clase social o de su pensamiento político o que desde su diversidad les servían económica y políticamente sin protestar. Las y los rebeldes y revolucionarios no tenían lugar en esa repartija de prebendas" (Paredes, 2014: 59).
} 
"La comunidad está constituida por mujeres y hombres como dos mitades imprescindibles, complementarias, no jerárquicas, recíprocas y autónomas una de la otra. Lo cual no necesariamente significa una heterosexualidad obligatoria, porque no estamos hablando de pareja, sino de par de representación política, no estamos hablando de familia, sino de comunidad” (Paredes, 2014: 87).

Puedo decir entonces que son dos las vertientes de diferenciación enunciadas desde feminismo comunitario en relación con el feminismo que se identifica como hegemónico. En primer lugar, la construcción de la comunidad desde los cuerpos sexuados de varones y mujeres -aunque particularmente no considero que el feminismo hegemónico excluya a los varones en la construcción de las comunidades/sociedades, salvo cierto feminismo de la diferencia muy popular en la década de los años '70 pero que considero fue superado, o por lo menos no es tan popular como entonces-.

La verdadera diferencia entre ambas posturas es, a mi modo de ver, la contundente crítica al neoliberalismo, desde el feminismo comunitario, que se plantea disidente, pero se reconoce feminista. Tal como plantea Julieta Paredes, “desneoliberalizar el género" se reconoce como una demanda de los feminismos del Sur, los cuales, ubicados geográficamente en tiempo y espacio, denuncian las relaciones de poder norte-sur como injustas e influyentes en las relaciones de intergéneros, aunque reconocen que el Patriarcado es más antiguo que el neoliberalismo.

\section{Reflexiones finales}

Necesariamente debo decir unas palabras en relación a la cuestión de la voz propia, y de la demanda del feminismo comunitario en relación a no ser habladas por otras. No es mi intención aquí más que presentar algunas características del feminismo comunitario como feminismo desde el Sur, que se reconoce feminista y se diferencia de un feminismo hegemónico que no reconoce sus demandas.

Como apuntaba previamente, la contribución del feminismo comunitario al movimiento feminista -en el cual se inscribe y diferencia a la vez-consiste en la ampliación del sujeto y objeto de los feminismos, construyendo así un sujeto reivindicativo plural: las mujeres; y un movimiento plural: los feminismos (plural). El feminismo comunitario propone una deconstrucciónconstrucción de la nueva sujeta política del/ de los feminismo(s), a la que refiere Lorena Cabnal:

"El feminismo que se está construyendo aboga por una deconstrucción del sujeto de mujer subordinada, sujeto apolítico, para pasar a un sujeto nuevo, liberador: la sujeta política, pensante y actuante, otra dimensión que conllevará a la mujer libre de condicionamientos de subalternidad histórica. "Reconocernos como sujetos y sujetas con derecho epistémico para crear pensamiento propio y con ello ir estableciendo nuevos paradigmas que nos permitan trascender las opresiones" (Robles Santana, n.d.: 15). 
La emergencia de estas voces ayuda a visibilizar la heterogeneidad del feminismo, porque como dice Francesca Gargallo "Como mujer blanca yo vivo sin conciencia los privilegios que el sistema racista me ha reservado desde la infancia. Están tan interiorizados y normalizados que no me percato de ellos, y, por ende, me abrogo el derecho de no reconocerlos, a menos que alguien me los señale" (citada por Robles Santana, n.d.: 2).

El feminismo comunitario es parte integrante del feminismo, se reconoce feminista y amplía las demandas de éste, por lo que su disidencia es parcial. Si pensamos en la emergencia de este feminismo dentro del movimiento, podemos rescatar las apreciaciones de Tapia cuando dice:

“[...] los movimientos sociales suelen hablar de algo que no tiene lugar en la sociedad, sobre la ausencia de algo deseable, cuya consecución se busca y conquista en el movimiento y en la reforma de los espacios políticos existentes" (Tapia, n.d.: 2), pudiendo reemplazar para este caso la palabra sociedad por la palabra feminismo. Es importante resaltar que la acción colectiva de los movimientos sociales, y entre ellos el feminismo aun actualmente "desborda[n] los lugares estables de la política" (Tapia, n.d.).

El feminismo comunitario tiene el mismo objetivo que el feminismo hegemónico en estos tiempos enuncia: acabar con la socialización generizada, acabar con los roles masculinos y femeninos, y sobre todo acabar con la organización jerárquica que ubica a las mujeres y a todo aquello que no sea el modelo de masculinidad hegemónica como subalterno.

Quisiera terminar con una reflexión en torno a la identidad como articulador de las demandas del colectivo de mujeres. En relación con la identidad y las identificaciones, y la evidente necesidad de problematizar los esencialismos, aunque comparto que no existe una esencia de lo que es ser-mujer, y que los esencialismos proponen siempre exclusiones, sí comparto la noción de que las mujeres, por más que en las diferentes posiciones de sujeto que ocupamos (Mouffe, 2007) podemos ser atravesadas por múltiples relaciones de subordinación, sí existe una experiencia común a todas, que es la opresión de la que somos objeto por parte del sistema patriarcal (Beltrán Pedreira, 1994) y que "la subestimación de las diferencias de género[siempre] implican la exclusión de las mujeres del mundo público" (Greblo, 2002: 175).

Sin embargo, aún destacando la particularidad del feminismo comunitario como movimiento dentro del movimiento, sigo pensando que todos los feminismos tienen el punto común de lucha contra el Patriarcado que nos oprime, y de hecho esto es algo afirmado desde el propio feminismo comunitario también. La existencia de voces que reivindiquen feminismos ${ }^{10}$, y no feminismo, es incluso coherente con el propio movimiento, y desde mi punto de vista, esta emergencia no divide sino que amplía la lucha y complejiza las identificaciones.

${ }^{10} \mathrm{La} \mathrm{S}$ en mayúscula es intencional para remarcar la pluralidad. 


\section{BIBLIOGRAFÍA}

- Arditi, Benjamín (2004): “Trayectoria y potencial político de la idea de sociedad civil”. En: Revista Mexicana de Sociología, vol. 66, nº. 1, pp. 1-21.

- Beltrán Pedreira, María Elena (1994): "Público y privado (Sobre feministas y liberales: argumentos en un debate acerca de los límites de lo político)". En: Doxa: Cuadernos de filosofía del derecho, vol. 1, no. 15-16, pp. 389-406.

- Forez Florez, Juliana (2005): “Aportes poscoloniales (latinoamericanos) para el estudio de los movimientos sociales". En: Tabula Rasa, vol. 3, pp. 73-96.

- Hartmann, Heidi (1980): "Un matrimonio mal avenido, hacia una unión más progresiva entre feminismo y marxismo”. En: Zona Abierta, vol. 24, pp. 85-113.

- Greblo, Edoardo (2002): Democracia. Léxico de política. $1^{\circ}$ Ed. Buenos Aires: Nueva Visión.

- Mouffe, Chantal (2007): En torno a lo político. Buenos Aires: FCE.

- O'Donnell, Guillermo (1978): “Apuntes para una teoría del Estado”. En: Revista Mexicana de Sociología, vol. 40, nº 4, pp. 1157-1199.

- Paredes, Julieta (2014): Hilando fino. Desde el feminismo comunitario. $2^{\circ} \mathrm{Ed}$. México: Creativecommons.

- Parra, Marcela (2005): "La construcción de los movimientos sociales como sujetos de estudio en América Latina". En: Athenea Digital nº 8, otoño de 2005, pp. 72-94. Disponible en: http://www.academia.edu/6625315/La_construcci\%C3\%B3n_de_los_movimientos_sociales_como_ sujetos_de_estudio_en_Am\%C3\%A9rica_Latina [26/04/2017].

- Ramos Rollón, María Luisa (1997): "La dimensión política de los movimientos sociales: algunos problemas. conceptuales”. En: REIS, nº. 79, pp. 247-263.

- Revilla Blanco, Marisa (1996): "El concepto de movimiento social: acción, identidad y sentido". En: Última Década, no. 5, pp. 1-18.

- Robles Santana, Aránzazu (n.d.): "Los feminismos comunitarios en AbyaYala. Una aproximación".

Disponible

en:

https://horizontesdecompromiso.files.wordpress.com/2013/01/los_feminismos_comunitarios.pdf [26/04/2017].

- Rubio García, Ana (2004): "Perspectivas teóricas en el estudio de los movimientos sociales". En: Circunstancia: revista de ciencias sociales del Instituto Universitario de Investigación Ortega y Gasset, $\mathrm{n}^{\mathrm{o}}$. 3. Disponible en: http://www.ortegaygasset.edu/publicaciones/circunstancia/ano-i--numero-3---enero-2004/estados-de-la-cuestion/perspectivas-teoricas-en-el-estudio-de-losmovimientos-sociales [26/04/2017].

- Tapia, Luis (n.d.): "Movimientos sociales, movimientos societales y los no lugares de la política". Disponible en: http://bibliotecavirtual.clacso.org.ar/ar/libros/secret/cuadernos/17/17tapia.pdf [26/04/2017].

- Valobra, Adriana (2015): "El Estado y las mujeres, concepciones en clave feminista". En: Estudios Sociales del Estado - vol. 1, $\mathrm{n}^{\mathrm{o}}$. 2, pp. 32-57. Disponible en: http://www.estudiossocialesdelestado.org/index.php/ese/article/view/44/33 [26/04/2017].

- Viguera, Anibal (2009): "Movimientos sociales y lucha de clases". En: Revista Conflicto Social, año $2, \mathrm{n}^{\circ} .1$, pp. 7-25. 